\title{
DEPENDÊNCIA QUÍMICA E GÊNERO: UMA LEITURA DA EXPERIÊNCIA FEMININA NA DROGADIÇÃO
}

\author{
Érica Henrique Ribeiro-Andrade \\ Mestre em Cognição e Linguagem. Especialista em Psicanálise. Especialista em Psicopedagogia. \\ Psicóloga. Docente dos cursos de Psicologia, Pedagogia e Educação Física do ISECENSA/ Campos- \\ RJ. \\ ericahribeiro@yahoo.com.br
}

Maria Francisca Teresa Barbosa Barreto

Graduanda do $3^{\circ}$ Período de Psicologia/ ISECENSA/ Campos-RJ

barbosamariateresa@hotmail.com

\section{Maria Luíza Mota}

Graduanda do $3^{\circ}$ Período de Psicologia/ ISECENSA/ Campos-RJ

Marialuizamota6@hotmail.com

\section{Maria Luiza Gomes Terra}

Graduanda do $3^{\circ}$ Período de Psicologia. / ISECENSA/ Campos-RJ

marialuizagomesterra@yahoo.com.br

\section{RESUMO}

No que diz respeito à experiência feminina na drogadição entende-se que certas particularidades tendem a tornar o sofrimento uma questão diversa daquela decorrente do uso que os homens fazem das drogas. A presente pesquisa objetivou refletir sobre o fenômeno adictivo na experiência feminina. A metodologia utilizada envolve características de uma pesquisa exploratória visando o levantamento bibliográfico como método de investigação, tendo a data de publicação das obras o período de 2010 a 2015. Este trabalho apresenta como resultados conceituações dos termos "dependência química" e "Gênero", tendo mantido uma ênfase ao gênero feminino. Foi possível elencar aspectos que diferenciam a experiência feminina na drogadição daquilo que ocorre com os homens dependentes químicos, dentre estes uma diferença no metabolismo dos corpos diante das substância e uma diferença quanto ao tipo de droga preferido. Os estudos apontaram que na lista dos motivadores e das conseqüências do uso abusivo de drogas, os fatores emocionais tendem a colocar a mulher numa posição de maior vulnerabilidade. A relação da sociedade com a drogadição feminina mostrou-se diversa da relação que a mesma estabelece com as adicções masculinas. Sugere-se que a produção científica possa intensificar a clarificação destas especificidades, no esforço para propiciar intervenções desejáveis.

Palavras-chave: Drogas; Mulher; Adicção; Psicologia; Saúde Pública.

Persp. online: hum. \& sociais aplicada., Campos dos Goytacazes, 16 (6), 69-76, 2016 


\title{
CENTRO EDUCACIONAL NOSSA SENHORA AUXILIADORA \\ INSTITUTOS SUPERIORES DE ENSINO DO CENSA CENTRO DE PESQUISA E PÓS-GRADUAÇÃO
}

\begin{abstract}
The women's experience with chemical dependency implies some particulars that turn the suffering in question different from men's suffering when they use drugs. The objective of research is reflect on the drug addiction phenomenon in feminine experience. The methodology utilized has the bibliography survey like method of investigation. Therefore, it involves characteristic of exploratory research. The works utilized have date of publication from 2010 to 2015. The results of research present concepts of "chemical dependency" and "Gender", with emphasis in the female gender. The aspects that differentiate the women's experience drug addiction what checks with chemical dependents men were related, among them one difference in the bodies metabolism when are in the effect of the substances and one difference as for the favorite kind of drugs.

The studies pointed that list of motivator and consequences of abusive use of drugs, the factors tend to put the woman in a position of greater vulnerability. The relationship of society with female drug addiction seemed different of relationship that the same has with men's drug addiction. Its is suggested the scientific production can intensify the clarifying the specificities for provide even more interventions.
\end{abstract}

Keywords: Drugs; Woman; Addiction; Psychology; Public Health.

\section{INTRODUÇÃO}

Os serviços de saúde apontam para o crescente prejuízo que a questão da dependência química tem trazido aos mais variados segmentos da sociedade. O fenômeno da drogadição resulta em um efeito deletério nos vários aspectos da vida do sujeito, ocasionando em pouco tempo problemas de ordem física, emocional e social. No que diz respeito a experiência feminina na drogadição entende-se que certas particularidades tendem a tornar o sofrimento em questão diverso daquele decorrente do uso que os homens fazem das drogas. Neste sentido, a presente pesquisa encontra o seu lugar juntamente com aquelas que se ocupam de produzir conhecimento sobre $o$ universo feminino e também com aquelas que têm a drogadição como foco, um problema que precisa ser urgentemente administrado tendo a reflexão e a pesquisa como aliadas.

Para Wole e Zilberman (2012) é notável que grande parte dos dependentes de drogas dos mais variados tipos é do gênero masculino, mas reconhecem que não se pode negar que nos últimos anos algo diferente tem ocorrido no consumo de substâncias psicoativas entre as mulheres.

Esses e outros motivos levaram Gomes (2010) a concordar que a dependência química em mulheres ainda é um campo de enigmas e que, portanto, precisa ser desvendado por meio da pesquisa científica.

Numa perspectiva geral objetivou-se nesta pesquisa refletir acerca das especificidades do fenômeno adictivo na experiência feminina. Especificamente conceituaram-se os eixos temáticos "dependência química", e "Gênero", numa ênfase ao gênero feminino. Intencionava-se também identificar na literatura apontamentos já existentes sobre a experiência adictiva feminina.

\section{METODOLOGIA}

Persp. online: hum. \& sociais aplicada., Campos dos Goytacazes, 16 (6), 69-76, 2016 


\section{CENTRO EDUCACIONAL NOSSA SENHORA AUXILIADORA \\ INSTITUTOS SUPERIORES DE ENSINO DO CENSA CENTRO DE PESQUISA E PÓS-GRADUAÇÃO}

A metodologia utilizada envolveu características de uma pesquisa exploratória tendo o levantamento bibliográfico como método de investigação. Este trabalho apresenta como descritores "dependência química" e "Gênero", tendo mantido uma ênfase ao gênero feminino, no que diz respeito ao segundo termo. Apresenta também uma correlação entre as variáveis investigadas buscando identificar o que tem sido afirmado nos últimos anos sobre a experiência da mulher com o uso de drogas. Utilizou-se como critério de inclusão obras impressas e indexadas em língua portuguesa sobre os eixos temáticos durante os anos 2010 a 2015. A metodologia envolve ainda consulta a alguns sites oficias do Ministério da Saúde e do Ministério de Segurança Pública acerca das informações disponíveis e pertinentes aos objetivos da pesquisa.

\section{DESENVOLVIMENTO}

Fiore (2012) dispõe em seu texto algumas conceituações importantes para o presente estudo. Para o autor, quando utiliza-se o termo "drogas", numa perspectiva mais geral da terminologia, refere-se a um produto farmacológico. Ao passo que ao utilizar-se o termo "Drogas lícitas", a referência está sendo feita, geralmente, à produtos que em sua composição apresentam substâncias psicotrópicas sem uma prescrição médica oficial. Dentre elas o autor menciona o álcool, as mais variadas bebidas estimulantes (café, chá e energéticos) e o cigarro de tabaco.

Para Pilon e Vasters (2011) é possível manter com as drogas diferentes maneiras de relação sem que necessariamente o sujeito que as experimente desenvolva um quadro de dependência. Os autores apontam também para uma diversidade de sentidos e motivações para que uma primeira experiência com as drogas ocorra.

Segundo Varalda e Cordeiro (2011), não existe uma sociedade sem a presença do uso de drogas, uma vez que o uso de substâncias que podem alterar o estado da consciência não pode ser contado em um período específico da história. Nos dias atuais a drogadição constitui-se em um problema de saúde pública em todo o mundo, no entanto, acreditam os autores, que no século passado, já entendia-se que o uso de drogas tinha um status de problema social, destacando que na juventude o consumo de drogas tornou-se parte dos diagnósticos mais comuns, envolvendo distúrbios crônicos, recorrentes e multifatoriais.

O Brasil desenvolve inúmeras pesquisas sobre a drogadição por meio de órgãos oficiais como o Centro Brasileiro de Informações sobre Drogas Psicotrópicas (CEBRID). Segundo dados do referido centro de pesquisas, não é possível determinar especificamente um fator que leva o sujeito ao uso de drogas, uma vez que são inúmeras as variáveis que podem levar a esta escolha. Dentre elas destaca-se desde a procura de alívio para dores físicas, angústias, medos e ansiedades, até a busca de mais satisfação e prazer por meio das sensações (Cebrid, 2010).

De acordo com Basthi (2011) o termo "Gênero" é uma categoria de análise relacional que nos permite observar as relações sociais em suas mais diversificadas proporções, honrando os múltiplos modos de ser feminino e masculino fora da definida divisão biológica e única entre os sexos.

Segundo Teixeira (2010) as diferenças de gênero se dão como construções sociais. Para o autor, a definição de gênero é diversa ao biológico e se direciona aos aspectos culturais. Desta forma, manter um estudo sobre o comportamento de homens e mulheres, demanda uma distância entre definições de sexo e gênero, e as questões de desigualdade implícitas nesta relação. Nesta direção Basthi (2011, p.19) afirma que "a igualdade de

Persp. online: hum. \& sociais aplicada., Campos dos Goytacazes, 16 (6), 69-76, 2016 


\section{CENTRO EDUCACIONAL NOSSA SENHORA AUXILIADORA \\ INSTITUTOS SUPERIORES DE ENSINO DO CENSA CENTRO DE PESQUISA E PÓS-GRADUAÇÃo}

tratamento e de oportunidades entre homens e mulheres - e, sobretudo, quanto às populações negra e indígena, com destaque para as mulheres negras e indígenas - ainda está longe de ser uma realidade.”.

Para Cyfer (2010), com Simone de Beauvoir e Gayle Rubin, o feminismo vinculou o conceito de que a identidade feminina não é uma variável inteligível resultante da biologia, mas sim uma situação, partindo de um apanhado ao longo da vida na ligação com o outro. Segundo a autora, as considerações a respeito da igualdade de gênero passariam a valorizar uma idéia de identidades que se constroem culturalmente, numa concepção que está mais a frente do que uma essência inscrita na anatomia.

Para Ilha, Leal e Soares (2010) a violência contra a mulher é um fenômeno que evidência as desigualdades entre mulheres e homens na sociedade. Para os autores trata-se de um problema de Saúde Pública, já que relaciona a integridade corporal e o estado psíquico e emocional da vítima, trazendo sérias e graves consequências para o seu pleno e completo desenvolvimento, e um prejuízo para o exercício da cidadania e dos direitos humanos.

O Relatório mundial sobre drogas publicado em 2015 pelo Escritório das Nações Unidas sobre Drogas e Crimes (UNODC), relata que a prevalência do uso de drogas continua frequente no mundo. De acordo com a pesquisa, estima-se que 246 milhões de pessoas com idade entre 15 e 64 anos tenha utilizado drogas ilícitas em 2013. O referido documento aponta que a produção, o comércio e o consumo de drogas envolvem tanto a saúde quanto os problemas sociais que englobam todas as raças, religiões, classes sociais, escolaridades e sexos. $\mathrm{O}$ consumo de drogas pela população feminina, apesar de pouco discutida, tem sofrido grande aumento nos últimos anos.

Souza, Oliveira e Nascimento (2014), percebem que a questão da dependência química entre as mulheres não tem recebido grandes investimentos de pesquisa em território Nacional. Os autores destacam a existência de estudos atribuindo esta realidade a uma relação de gênero desigual. Identificam também que estudos recentes tem considerado a iniciativa autônoma da mulher em seu envolvimento com as drogas.

Ao se referir ao cigarro, LION (2013) destaca a vulnerabilidade das mulheres com o tabagismo. A comunidade científica, afirma o autor, tem buscado relacionar a diferença do metabolismo sobre a nicotina entre ambos os sexos evidenciando que as mulheres apresentam um metabolismo mais rápido devido a vários processos enzimáticos que levam os níveis circulantes da nicotina a serem mais ativos.

Uma das especificidades femininas diante da drogadição implica o fato da mulher ser vitima do uso de drogas também por uma via indireta em função, muitas vezes, da existência de um companheiro drogadicto. Para Souza, Oliveira e Nascimento (2014) a relação entre o consumo de drogas e a pratica da violência é consenso na literatura, onde as mulheres são vistas como vítimas da violência física ou psicológica do companheiro que é usuário de drogas. Afirmam ainda que, a imagem da mulher, na mídia, é vista como dócil, cuidadora, ingênua, sendo assim a principal vítima do homem adicto com quem ela convive.

Pedroso (2013) menciona que em geral, as mulheres que apresentam um caso de dependência química possuem em sua família laços conflituosos envolvendo a falta de uma hierarquia definida, uma distância entre os membros e uma ausência de harmonia. Ou seja, um conjunto de características negativas que marcam a vida familiar destas mulheres.

Em sua pesquisa de dissertação de mestrado em Saúde Pública pela FIOCRUZ, Silva (2012) identifica pesquisas realizadas nestes últimos vinte anos, apontando que as mulheres alcoolistas podem apresentar um déficit na autoestima, acarretando dificuldades como a ansiedade, a desmotivação e distúrbios comportamentais.

Persp. online: hum. \& sociais aplicada., Campos dos Goytacazes, 16 (6), 69-76, 2016 


\section{CENTRO EDUCACIONAL NOSSA SENHORA AUXILIADORA \\ INSTITUTOS SUPERIORES DE ENSINO DO CENSA CENTRO DE PESQUISA E PÓS-GRADUAÇÃo}

Esta autora, tendo como base estudos como os de Pillon e Luiz(2004) e os de Nóbrega e Oliveira(2005), destaca também que as comorbidades, ou seja, dois ou mais problemas patogênicos simultâneos, que são mais frequentes nestas mulheres dependentes do álcool, são os transtornos ansiosos como fobia e estresse e os transtornos de humor como a depressão e mania. Já os homens apresentam transtornos significativos de personalidade como o transtorno anti-social.

Segundo Assunção (2015) a sociedade é altamente reprobatória quando a questão é o alcoolismo feminino. Explica que isso se dá devido ao desvio comportamental do que é socialmente aceito para o papel da mulher, negativando o ideário coletivo sobre sua identidade. Esta autora faz uma crítica acerca da forma impositiva pela qual a sociedade, a todo momento, tenta moldar a conduta feminina, propondo que novos estudos sejam feitos sobre como essas mulheres se veem em comparação de como são vistas pelo meio social.

De acordo com Silva (2012) o uso abusivo de bebidas alcoólicas afeta de maneira significativa os diversos papéis que são atribuídos às mulheres, em especial na maternidade, na vida familiar e no trabalho. Conforme essa autora, a pressão social que uma mulher sofre quando se torna uma usuária de droga é muito mais rigorosa do que se tratando de um homem.

Wolle e Zilberman (2011) acreditam que existem entre os motivos para o crescimento do consumo de drogas pela população feminina está a reconhecível alteração no estilo de vida das mulheres, como o seu crescimento no mercado de trabalho, o acúmulo de funções, o aumento da carga horária entre outros.

Souza (2013) aponta que levando em conta diversos mobilizadores para o uso, dentre os quais destaca-se a depressão, a ansiedade e os padrões de beleza exigidos pela sociedade, o consumo de drogas pode se tornar um adaptador para que a mulher sinta-se cumprindo suas obrigações sociais.

A autora afirma ainda que o relacionamento afetivo de mulheres com traficantes de drogas e o desejo pela manutenção desta relação, tem se mostrado como um fator importante para a entrada de mulheres no crime de contravenção. Além disso, uma ascendência hierárquica diante de outras mulheres e até homens, tem também contribuído para a entrada da mulher no tráfico, mantendo o ciclo de sua dependência ativo.

Dados do Departamento Penitenciário Nacional (DEPEN) confirmam tais idéias ao registrarem que além do aumento do consumo de drogas entre mulheres, nota-se um crescimento da entrada de mulheres nas penitenciárias femininas, sendo o tráfico um dos principais motivos para esse crescimento carcerário (BRASIL, 2014).

Segundo recentes pesquisas, a maconha, a anfetamina e a cocaína são três vezes mais utilizadas por homens do que por mulheres, ao passo que os tranquilizantes e os opióides são categorias de substâncias mais recorrentes entre as mulheres. Obteve-se também que, em determinados países, mulheres que tem o costume de injetar drogas estão mais vulneráveis à infecções por HIV do que homens (UNODC,2015).

Uma grande parcela de usuários de drogas continua a perder suas vidas prematuramente, afirma o Diretor Executivo da UNODC, Yuri Fedotov, que estima um total de 187.100 mortes por razões de drogas em 2013. Dentre estas mortes, infere-se um número significativo de mulheres, uma vez que, segundo o Diretor, as mulheres enfrentam mais barreiras para o tratamento (UNODC,2015).

Persp. online: hum. \& sociais aplicada., Campos dos Goytacazes, 16 (6), 69-76, 2016 


\section{CENTRO EDUCACIONAL NOSSA SENHORA AUXILIADORA \\ INSTITUTOS SUPERIORES DE ENSINO DO CENSA CENTRO DE PESQUISA E PÓS-GRADUAÇÃO}

Evidenciando a situação feminina com a dependência química, em relação aos homens drogadictos, podemos ressaltar que as mulheres tem mais problemas físicos e mais problemas familiares, além de o índice de adicção química feminina ter crescido estatisticamente nos últimos anos (LENAD, 2013).

\section{CONSIDERAÇÕES FINAIS}

Ao longo do levantamento identificou-se uma quantidade de produção científica considerada pequena sobre a relação das variáveis "gênero feminino" e "drogadição" nos últimos 5 anos. Esse dado contrasta com as afirmativas de que o índice de dependência química entre mulheres está aumentando. Os resultados do levantamento realizado permitiram o elencar de alguns aspectos que diferenciam a experiência feminina na drogadição daquilo que ocorre com os homens dependentes químicos.

A presente pesquisa deixa como ganho principal a confirmação da hipótese inicial que acreditava em contornos muito peculiares nas adicções femininas. Sugere-se que no âmbito da saúde e também no âmbito das ciências humanas e sociais aplicadas, a produção científica possa intensificar a clarificação destas especificidades, não para acentuar a desigualdade já existente, mas para propiciar intervenções ainda mais assertivas por parte dos familiares, amigos e profissionais pertinentes.

\section{REFERÊNCIAS}

ASSUNÇÃO, B. D. Estigmas, saúde mental e drogas: uma discussão sobre o alcoolismo feminino. Maceió,2015. . Disponível em: <http://eventos.livera.com.br/trabalho/98-1020489_30_06_2015_23-5806_8832.PDF>. Acesso em: 02 mar. 2016.

BASTHI, A. Guia para Jornalismo sobre: Gênero, Raça e Etnia. Federação Nacional dos Jornalistas (FNAJ), 2011.

BRASIL. Ministério da Justiça. Levantamento Nacional de Informações Penintenciárias INFOPEN Mulheres. 2014.

CEBRID - Centro Brasileiro de Informações sobre Drogas Psicotrópicas - Departamento de Psicobiologia UNIFESP, boletim 64-65, 2010.

CYFER, I. Liberalismo e feminismo: igualdade de gênero em Carole Pateman e Martha Nussbaum. Rev. Sociol. Polít., Curitiba, v. 18, n. 36, p. 135-146, jun. 2010.

FIORE, M. O lugar do Estado na questão das drogas: o paradigma proibicionista e as alternativas.Novos estud. CEBRAP, São Paulo , n. 92,p. 9-21,Mar 2012 . Disponível em:

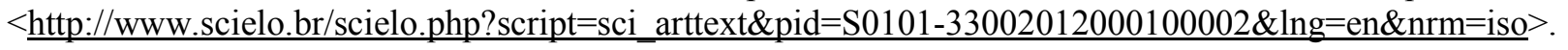
Acesso em: 11 nov. 2015.

GOMES, K.V. Dependência química em mulheres: figuras de um sintoma partilhado. Orientadora Maria Ines Assunção Fernandes. Tese de doutorado. USP. São Paulo, 2010.

Persp. online: hum. \& sociais aplicada., Campos dos Goytacazes, 16 (6), 69-76, 2016 


\section{CENTRO EDUCACIONAL NOSSA SENHORA AUXILIADORA \\ INSTITUTOS SUPERIORES DE ENSINO DO CENSA CENTRO DE PESQUISA E PÓS-GRADUAÇÃO}

ILHA, M. M. ; LEAL,S.M. C.; SOARES, J. S. F. . Mulheres internadas por agressão em um hospital de pronto socorro: (in)visibilidade da Violência. Rev Gaúcha Enferm., Porto Alegre (RS) 2010 jun;31(2):328-34.

LION, E. A.V. Impacto do tabagismo na saúde feminina em Aliança do controle do Tabagismo, 2013, p.4 http://actbr.org.br/uploads/conteudo/843 ACT saude feminina 2013.pdf. Acesso em 24/02/16

SILVA, M.G.B. . O pensar e o agir das mulheres assistidas em um centro de atenção psicossocial de álcool e outras drogas: alcoolismo feminino e o caminho para a recuperação. Dissertação Mestrado Profissional em Saúde Pública. Centro de Pesquisas Aggeu Magalhães, Fundação Oswaldo Cruz. Recife: 2012. file://CC:/Users/Maria $\% 20$ Tereza/Downloads/o\%20pensar $\% 20 \mathrm{e} \% 20 \mathrm{o} \% 20 \mathrm{agir} \% 20$ das $\% 20$ mulheres $\% 20$ drogadic tas.pdf Acesso em 02/03/16

SOUZA, M.R. R.; OLIVEIRA, J. F; NASCIMENTO, E. R.. A Saúde de mulheres e o fenômeno das drogas. Revistas brasileiras em Texto Contexto Enfermagem, Florianópolis, 2014 Jan- Mar; 23(1): 92- 100. http://www.scielo.br/pdf/tce/v23n1/pt 0104-0707-tce-23-01-00092.pdf. Acesso em 24/02/16

SOUZA, M.R.R. Repercussões do envolvimento com drogas para a saúde de mulheres. CAPSAD SalvadorBA 2013. file:///C:/Users/Maria\%20Tereza/Downloads/mono\%20mulher\%20e\%20drogas.pdf Acesso em $02 / 03 / 16$

TEIXEIRA, A.B.M. Gêneros e práticas culturais: desafios históricos e saberes interdisciplinares [online]. Campina Grande: EDUEPB, 2010. http://static.scielo.org/scielobooks/tg384/pdf/machado-9788578791193.pdf

PEDROSO, D. T. Meninas no crack: Vulnerabilidade ao Uso e Dependência em Revista de Psicologia da
IMED,
Jul.-
Dez,
2013,
5 ,
n. 2 ,
p.
$126-132$

http://webcache.googleusercontent.com/search?q=cache:6TCPkCK8zKEJ:dialnet.unirioja.es/descarga/articulo/5 155023.pdf $+\& \mathrm{~cd}=1 \& \mathrm{hl}=\mathrm{pt}-\mathrm{BR} \& \mathrm{ct}=\mathrm{clnk} \& \mathrm{gl}=$ br. . Acesso em 24/02/16

PILLON, S. C.; VASTERS, G. P.. O uso de drogas por adolescentes e suas percepções sobre adesão e abandono de tratamento especializado Rev. Latino-Am. Enfermagem, vol.19, mar-abr, 2011. $\leq$ http://www.scielo.br/pdf/rlae/v19n2/pt 13.pdf. Acesso em 11/11/2015>.

VARALDA, R. B.; CORDEIRO, F. A. Crack: acolher é reconstruir vidas. Revista Jurídica Consulex, Brasília, ano XV, n. 352, p. 24-25, set. 2011.

WOLlE, C. C.; ZILBERMAN, M. L. In: DIEHL A., CORDEIRO, D.C. \& LARANJEIRA, R. (org.). Dependência Química: prevenção, tratamento e políticas públicas. Porto Alegre: Artmed, 2011, p: 375-382. 


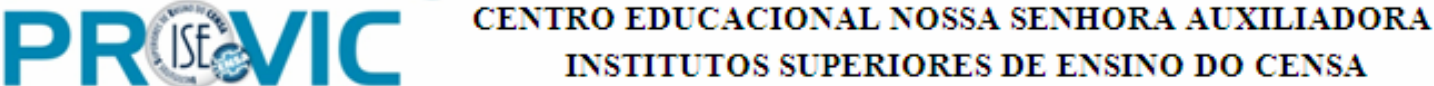 \\ PROGRAMA VOLUNTÁRIO DE INICLACÃo CIENTÍFICA \\ CENTRO DE PESQUISA E PÓS-GRADUAC̣̃̃o}

http://inpad.org.br/ lenad-familia/ Acesso em 26/02/16

http://inpad.org.br/wp-content/uploads/2013/11/Familia_Nov.pdf Acesso em 26/02/16

https://www.unodc.org/lpo-brazil/pt/frontpage/2015/06/relatorio-mundial-sobre-drogas-de-2015--o-uso-dedrogas-e-estavel--mas-o-acesso-ao-tratamento-da-dependencia-e-do-hiv-ainda-e-baixo.html Acesso em 03/03/16

http://www.unodc.org/documents/wdr2015/World_Drug_Report_2015.pdf Acesso em 04/03/16

http://www.unodc.org/wdr2015/field/WDR15 Methodology.pdf

Persp. online: hum. \& sociais aplicada., Campos dos Goytacazes, 16 (6), 69-76, 2016 